\title{
Erratum to: Efficacy and safety of aprepitant for the prevention of chemotherapy-induced nausea and vomiting during the first cycle of moderately emetogenic chemotherapy in Korean patients with a broad range of tumor types
}

\author{
Jeong Eun Kim ${ }^{1}$ • Joung-Soon Jang ${ }^{2}$. Jae-Weon Kim ${ }^{3}$ • Sung Yong Lee ${ }^{4}$. \\ Chi-Heum Cho ${ }^{5}$ Myung-Ah Lee ${ }^{6}$ - Do-Jin Kim ${ }^{7} \cdot$ Myung-Ju Ahn ${ }^{8} \cdot K^{2}$ Yll Yeon Lee ${ }^{9}$. \\ Sun Jin Sym ${ }^{10}$ - Myong Choel Lim ${ }^{11}$ • Hun Jung ${ }^{12}$ Cho Eun Kim ${ }^{12} \cdot$ Kyung Wan Min ${ }^{12}$
}

Published online: 14 February 2017

(C) Springer-Verlag Berlin Heidelberg 2017

\section{Erratum to: Support Care Cancer (2017) 25:801-809 DOI 10.1007/s00520-016-3463-0}

Unfortunately, the original version of this article contained errors.

The names of Sung Yong Lee and Cho Eun Kim were incorrectly captured and are now corrected in this article. Affiliation 12 is also corrected. The baseline demographics table (Table 2) incorrectly reported percentages calculated

The online version of the original article can be found at http://dx.doi.org/ 10.1007/s00520-016-3463-0.?

Joung-Soon Jang

alsaba@hanmail.net

1 Department of Oncology, Asan Medical Center, University of Ulsan, College of Medicine, 88 Olympic ro 43 gil, Sonpa-gu, Seoul, South Korea

2 Department of Internal Medicine, Chung-Ang University College of Medicine, 101 Heukseok-ro, Dongjak-gu, Seoul, South Korea

3 Department of Obstetrics and Gynecology, Seoul National University College of Medicine, 101 Daehak-ro Jongno-gu, Seoul, South Korea

4 Division of Respiratory and Critical Care Medicine, Department of Internal Medicine, Korea University College of Medicine, 73 Inchon-ro, Seongbuk-gu, Seoul, South Korea

5 Department of Obstetrics and Gynecology, School of Medicine, Keimyung University, 56 Dalseungro Jungku, Daegu, South Korea

6 Department of Medical Oncology, Seoul St. Mary's Hospital, The Catholic University of Korea, 901 Hyehwa-dong, Jongno-gu, Seoul, South Korea out of the total population in each chemotherapy group, instead of out of the total population (as with the rest of the table). Below are the correct percentages:

\begin{tabular}{lll}
$\begin{array}{l}\text { Chemotherapeutic regimen, } \\
\mathrm{n}(\%)\end{array}$ & Aprepitant $(n=237)$ & Control $(n=243)$ \\
Carboplatin-based & $156(65.8)$ & $156(64.2)$ \\
Oxaliplatin-based & $67(28.3)$ & $69(28.4)$ \\
Irinotecan-based & $14(5.9)$ & $18(7.4)$ \\
\hline
\end{tabular}

Division of Allergy and Respiratory Medicine, Department of Internal Medicine, Soonchunhyang University Bucheon Hospital, Soonchunhyang University College of Medicine, 170 Jomaru-ro, Wonmi-gu, Bucheon, South Korea

8 Division of Hematology-Oncology, Department of Medicine, The Samsung Medical Center, Sungkyunkwan University School of Medicine, 135-710 Irwon-dong, Gangnam-gu, Seoul, South Korea

9 Department of Surgery, Kyung Hee University School of Medicine, 26 Kyungheedae-ro, Dongdaemun-gu, Seoul, South Korea

10 Division of Hematology and Medical Oncology, Department of Internal Medicine, Gachon University Gil Medical Center, Incheon Regional Cancer Center, Gachon University School of Medicine, 1198 Guwol-dong, Namdong-Gu, Incheon, South Korea

11 Gynecologic Cancer Branch, Research Institute and Hospital, National Cancer Center, 323 Ilsan-ro, Ilsandong-gu, Goyang-si, Gyeonggi-do, South Korea

12 Merck Sharp \& Dohme, Seoul City Credit Building, 163, Mapo-daero, Mapo-gu, Seou, South Korea 